\title{
Voriconazole Treatment for Subacute Invasive and Chronic Pulmonary Aspergillosis
}

\author{
Helen Sambatakou, MD, ${ }^{a}$ Bertrand Dupont, ${ }^{b}$ Hartmut Lode, ${ }^{c, d}$ David W. Denning, FRCPe,f \\ ${ }^{a}$ Department of Medicine and Infectious Diseases, University of Athens, Greece; ${ }^{b}$ Institut Pasteur and Hopital Necker, Paris, France; \\ ${ }^{c}$ Department of Chest and Infectious Diseases, Hospital Heckeshorn, Berlin, Germany; ${ }^{d}$ Freie Universitat Berlin, Germany; ${ }^{e}$ The \\ University of Manchester, United Kingdom; ${ }^{f}$ Wythenshawe Hospital, Manchester, United Kingdom.
}

\begin{abstract}
BACKROUND: Voriconazole is a novel triazole antifungal with a broad spectrum including Aspergillus species. We conducted an open, noncomparative multicenter study to evaluate the efficacy and safety of voriconazole in subacute invasive and chronic pulmonary aspergillosis (CPA).

METHODS: Patients without profound neutropenia and a proven or probable diagnosis of subacute invasive aspergillosis (IA) or CPA received voriconazole $200 \mathrm{mg}$ twice daily for a period of 4-24 weeks as primary or salvage therapy. Dose escalation was allowed if efficacy was suboptimal, and toleration and safety were satisfactory. Response was assessed by clinical, radiological and mycological changes. A complete or partial response in subacute IA and improved or stable in CPA were assessed as favorable responses.

RESULTS: Of 39 patients treated, 36 were assessable. The majority of patients had subacute IA $(\mathrm{n}=21)$, proven in all 11 extra-pulmonary and in 23/25 (92\%) of the pulmonary cases. Voriconazole was given as primary therapy in $22(61 \%)$. All patients receiving salvage therapy $(n=14)$ had refractory IA, having failed itraconazole or amphotericin B $(\mathrm{AmB})$ or both. Overall, a complete or partial response was seen in $9 / 21(43 \%)$ of subacute IA and improved or stable in $12 / 15(80 \%)$ of those with CPA. Adverse events, mainly liver function test abnormalities, skin reactions, and visual disturbances were mild and transient, leading to early discontinuation of treatment in 5 cases.

CONCLUSIONS: In patients with subacute IA and CPA, voriconazole was efficacious as salvage or primary therapy. (C) 2006 Elsevier Inc. All rights reserved.
\end{abstract}

KEYWORDS: Aspergilloma; Aspergillus; Cerebral; Osteomyelitis; Respiratory

The incidence of invasive aspergillosis (IA) has increased among patients with neutropenia; neutrophil or macrophage dysfunction; bone marrow or solid organ transplant; or congenital or acquired immunodeficiency. ${ }^{1}$ The pattern of pulmonary aspergillosis is determined by the patient's immunological status and the condition of the lung. ${ }^{2}$ Angioinvasive disease is the form most often encountered in neutropenic patients. Equally lethal are acute invasive forms in other highly immunocompromised patients. Subacute invasive (chronic necrotizing) pulmonary aspergillosis has

Requests for reprints should be addressed to David W. Denning, Education and Research Centre, Wythenshawe Hospital, Southmoor Rd, Manchester M23 9LT, UK.

E-mail address: ddenning@manchester.ac.uk been described, usually in patients with acquired immune deficiency syndrome (AIDS), diabetes mellitus, chronic granulomatous disease, or low dose corticosteroid therapy. ${ }^{3}$ Among chronic forms of pulmonary aspergillosis (CPA), simple aspergilloma, chronic cavitary (CCPA) (complex aspergillomas), and chronic fibrosing pulmonary aspergillosis (CFPA) are described, in addition to allergic forms. Patients with CCPA often have innate immune defense defects (ie, mannose binding protein and cytokine abnormalities). ${ }^{4,5}$

CCPA is characterized by the expansion of single or multiple cavities over time or the development of new ones, some containing fungus balls. In some cases, extensive pulmonary fibrosis is predominant. ${ }^{6}$ The above characteristics are helpful in distinguishing CCPA from a single as- 
pergilloma. The incidence of CCPA is not known. Establishing a definite diagnosis requires a combination of serological, clinical, and radiological characteristic features, and not the approach taken with IA. ${ }^{3,6}$ For example, hyphae might be visualized within a cavity and not in lung tissue, and a biopsy reveals chronic inflammation and fibrosis. Positive precipitating Aspergillus antibodies in blood are almost always positive. It is characterized by an indolent progressive course lasting for years. Prospective treatment trials are lacking, and the optimal therapy and its duration is unknown. Surgery for CPA, other than simple aspergillomas, carries a high incidence of complications (eg, mortality, bronchopleural fistula, pleural space aspergillosis). ${ }^{6}$

Amphotericin B (AmB) has been used extensively in various forms of aspergillosis. In acute IA the overall response rate has been $\sim 35 \%$, with differing responses in patients with differing underlying disease, sites of infection, and duration of therapy. ${ }^{7,8}$ AmB lipid formulations have improved safety profiles, but there is no evidence of a superiority in efficacy. Moreover, A. terreus and A. nidulans are resistant to AmB. ${ }^{9-11}$ Caspofungin has proven useful as an alternative to $\mathrm{AmB}$, with a more tolerable safety profile. ${ }^{13}$ In CPA, AmB and caspofungin have a limited place because of the chronicity of infection. Itraconazole is an alternative, with response rates (complete/improved) of $39 \%$ and $63 \%$, respectively for IA, according to recent and earlier studies. ${ }^{13-18}$ Emergence of A. fumigatus resistant in vitro to itraconazole associated with in vivo failure has been reported. ${ }^{9,19}$

Voriconazole is a new broad-spectrum azole. Data from animal models showed vori to be effective treatment for pulmonary and disseminated aspergillosis in both immunocompetent and immunocompromised animal models. ${ }^{20,21}$ The bioavailability of oral voriconazole (up to $96 \%$ ) is better than that of itraconazole. ${ }^{22}$ A noncomparative study of acute IA as primary and salvage therapy demonstrated an overall response rate of $48 \% .^{23}$ In a randomized study of acute IA, a 12-week response rate of $53 \%$ with voriconazole primary treatment was superior to $\mathrm{AmB}(32 \%){ }^{24}$

\section{PATIENTS AND METHODS}

Protocol 150-303 was an open, noncomparative multicenter study of the efficacy and safety of voriconazole in chronic IA, given as primary or salvage therapy, conducted between July 1993 and November 1995 in 12 centers in Europe. The protocol was approved by each institutional ethical committee. Written informed concent was obtained from all patients.
Patients with definite or probable subacute IA at different body sites ( $>1$ month of disease before diagnosis) or CPA (>3 months disease before diagnosis) were enrolled. Patients had to be older than 18 years of age without profound neutropenia (ie, $>500 \times 10^{9} / \mathrm{L}$ ). Patients were ineligible if they had acute IA $(<1$ month of disease) or a noninvasive form of aspergillosis, such as allergic bronchopulmonary aspergillosis, or a simple aspergilloma without evidence of progressive disease, hypersensitivity, or intolerance to azole antifungals. Patients were also ineligible if they were receiving drugs that induce or inhibit hepatic cytochrome $\mathrm{P} 450$ with potential drug-drug interaction with voriconazole; were pregnant or lactating; had a life expectancy $<1$ month; or had aminotransferase, alkaline phosphatases and serum creatinine levels higher than 3,2 , and 1.5 times the upper limit of normal, respectively.

In patients previously treated with subtherapeutic doses of conventional antifungal treatment for $<10$ days, voriconazole was given as primary therapy. In cases where there was inadequate response or intolerance to the conventional antifungal therapy, a switch to voriconazole was allowed as a salvage therapy.

\section{Subacute Invasive Aspergillosis}

The certainty of diagnosis was based on established criteria $^{13,24}$ for patients with IA. Establishing a definite diagnosis of subacute IA required the use of an invasive procedure. It was defined as a clinically consistent disease plus a positive culture for Aspergillus spp. from tissue obtained by an invasive procedure, such as transbronchial biopsy or percutaneous needle aspiration, or hyphae consistent with Aspergillus spp. in a biopsy specimen or aspirate with or without a positive culture for Aspergillus from the same organ. Patients with clinically compatible disease and characteristic radiological findings not attributable to other factors with supportive evidence of infection such as recovery of Aspergillus spp. from respiratory fluids were also classified as probable cases according to established criteria ${ }^{14}$ and applied only to patients with pulmonary infection.

\section{Chronic Pulmonary Aspergillosis}

Patients enrolled had evidence of pulmonary aspergillosis infection for at least 3 months, with characteristic radiology (cavitation which was progressive, without a fungus ball) and positive mycology. Many also had positive Aspergillusprecipitating antibody in the blood, although this was not required in the protocol. Since the study was completed, a change in classification of these patients' disease has occurred, ${ }^{6}$ and the classification provided in this report is based on this. The term CPA is used here to denote nonal- 
lergic cavitary pulmonary aspergillosis, excluding simple aspergillomas. In all cases, there was inadequate response or intolerance to the conventional antifungal therapy.

\section{Treatment and Study Procedures}

Voriconazole was given orally in an initial dose of $200 \mathrm{mg}$ twice daily. In patients not responding to the initial dose schedule and with good tolerability, dose escalation to 250 mg BID was allowed. The duration of study treatment was 4-24 weeks, depending on the overall response of the patient according to investigator's judgment. No concurrent systemic antifungal therapy was allowed during voriconazole treatment. Data concerning underlying disease, immunocompromising factors, concomitant medications, as well as laboratory data were collected at baseline and at regular intervals. Response to voriconazole was assessed on patients' clinical, radiological, and mycological improvement at regular intervals and the end of treatment. Tolerability and safety was assessed by careful monitoring of any clinical and laboratory adverse event. Follow-up evaluation was at 4 and 12 weeks after the end of treatment. Sera for aspergillus antigen were collected and tested retrospectively (Platelia, BioRad, Edmonds, Wash).

\section{Case Review}

All case report forms and available radiology were carefully reviewed by a single reviewer (D.W.D.), and diagnostic criteria and outcome were both re-evaluated based on the available data. The patients were classified according to the site of involvement. The majority of patients had only one site of aspergillosis; however, in some cases there was concomitant sinus and cerebral disease, extension of pulmonary aspergillosis into a thoracic wall abscess, or disseminated disease at the time of enrolment. In cases with more than one site involved, the predominant site was the one with proven diagnosis, whereas the other site was classified as probable.

Responses of subacute IA were categorized as complete, partial, stable, failure or not evaluable, according to the Mycoses Study Group criteria. ${ }^{13}$ Complete response was defined by the resolution of all signs and symptoms, nearly complete resolution of radiological findings, and other supportive evidence (mycology). Partial response was defined as a clinically meaningful improvement and $>50 \%$ improvement in radiology findings. A response was characterized as stable in cases of minor or no improvement in attributable signs and symptoms and $<50 \%$ radiologic improvement. When short courses of therapy did not allow an assessment of outcome or in cases of death unrelated to IA without evidence of deterioration of fungal infection a stable response was recorded. Failure was determined as deterioration in attributable clinical, radiographic abnormalities necessitating alternative antifungal therapy or death due to IA. Complete and partial responses were classified as favorable outcomes.

For CPA cases, however, the outcome was defined as improved, stable or failure, as described previously. ${ }^{6}$ To achieve an improved response, there had to be marked improvement in patient's symptoms and signs (including weight gain, if previously lost), stabilization or improvement in radiological abnormalities, and negative fungal cultures. Relapse was also determined during posttreatment follow-up.

\section{RESULTS}

A total of 39 patients were recruited in the study over the 3 -year study period. Three patients were excluded because inadequate data supported the diagnosis of aspergillosis. Ten patients were women, and $26(72 \%)$ were men. The patients' ages ranged from 12 to 93 years (median 55 years) and weight from 26 to $110 \mathrm{~kg}$ (median $60 \mathrm{~kg}$ ). There was a wide variety of underlying conditions and immunological status (Table 1). Patients were classified as acute or subacute IA or CPA. None was neutropenic $\left(<500 \times 10^{6}\right.$ cells/L), with the exception of an AIDS patient with transient neutropenia. In some cases, more than one predisposing factor for IA was present.

The most common site of infection was the lungs, accounting for 25 cases $(69.4 \%)$. Fever was not a predominant symptom, in contrast to acute forms of aspergillosis, and was present in $9 / 35$ (26\%) patients. All the nonpulmonary IA cases had a definite diagnosis. Of the 25 patients with pulmonary aspergillosis, 23 (92\%) had a definite and 2 a probable IA diagnosis, both subacute IA. Of the $15 \mathrm{CPA}$ patients, 14 presented with multi-cavitary disease and, in one case, bilateral upper lobe fibrosis and pleural disease. Nine patients had a cavity containing an aspergilloma and 5 patients had no aspergilloma in the cavities. In 2 patients, an Aspergillus empyema was present. The right upper lobe was the most common site of involvement (11 cases), whereas left upper lobes were involved in 6 cases, and left lower lobes were involved in 1 case. Fibrosis and pleural thickening coexisted in many cases, along with the cavity formation and expansion of the cavity over time.

Multiple respiratory samples and more than one modality (sputum, BAL sample, brushing, transbronchial biopsy, operative specimen) were used to establish the diagnosis. Mycological confirmation was achieved in all cases by histology, direct microscopy or culture or combination. $A$. fumigatus was cultured from 27 patients, whereas A. niger, A. candidus, and A. terreus, A. flavus, from 1 each. In the CPA cases, A. fumigatus predominated, but mixed cultures of A. flavus and A. candidus, both with A. fumigatus, were revealed from a pleural aspirate and sputum, respectively, in two cases. Four Aspergillus isolates were not speciated. From 6 biopsy specimens, histology was positive, but culture was not done in 4 cases and was negative in 2 cases. Aspergillus antibodies were positive in all CPA patients in whom the result was recorded. Aspergillus antigen testing $(\mathrm{OD} \geq 0.5)$ was positive at some time on or after enrollment in 14 of $22(64 \%)$ of those tested.

Voriconazole was given as a primary therapy in 22 (61\%) of 36 patients. The other 14 cases had failed prior 
Table 1 Underlying Disease/Condition of Patients with Subacute IA and CPA, Categorized by the Underlying Factor Perceived to be the Most Important for the Development of Aspergillosis

\begin{tabular}{lcl}
\hline Underlying Disease/Condition & No. (\%) of Patients & Site and Pattern of Involvement \\
\hline Immunocompromised & $14(39)$ & \\
AML & 2 & Subacute pulmonary IA, spine osteomyelitis \\
Allogeneic BMT with GVHD & 2 & Sinusitis, cerebral \\
AIDS & 6 & 5 subacute pulmonary, 1 laryngitis/tracheitis \\
Heart transplant & 1 & Osteomyelitis of sternum \\
CGD & 2 & Subacute pulmonary IA \\
ITP & 1 & Subacute pulmonary IA \\
Pulmonary disease only & $11(30.5)$ & \\
COPD* & 2 & CPA \\
Prior pulmonary TB $\dagger$ & 7 & CPA \\
Bronchiectasis & 2 & CPA \\
Other & $11(31)$ & \\
Diabetes mellitus & 4 & 1 subacute IA, 3 sinusitis \\
Alcoholism $\ddagger$ & 1 & CPA \\
Post-traumatic & 1 & Osteomyelitis of carpal bones \\
Post-operative & 2 & Cerebral both \\
ESRD & 1 & CPA \\
Ankylosing arthritis & 1 & CPA \\
None & 1 & CPA \\
\hline
\end{tabular}

$\mathrm{AML}=$ acute myeloid leukemia; $\mathrm{BMT}=$ bone marrow transplant; $\mathrm{GVHD}=$ graft-versus-host disease; $\mathrm{CGD}=$ chronic granulomatous disease; ITP $=$ idiopathic thrombocytopenic purpura; COPD = chronic obstructive pulmonary disease; $T B=$ tuberculosis; $E S R D=$ end-stage renal disease, secondary to surgically resected kidney due to renal cell carcinoma.

*Plus empyema in one case.

$\dagger$ Including atypical mycobacterial disease with COPD, emphysema, chronic alcoholism in one case each.

$\ddagger$ Plus COPD.

antifungal therapy; $8 / 14(57.1 \%)$ were refractory cases to more than one antifungal. The duration of prior treatment in salvage cases ranged from 11 days to 18 months. Infections had failed to respond to $\mathrm{AmB}$ in 6, itraconazole in 14 patients, and liposomal $\mathrm{AmB}$ and flucytosine in 1 patient each. AmB was given intrapleurally in one case.

Voriconazole was given orally for periods ranging from 4 to 411 days (median 108 days). Early discontinuation was recorded in 11 patients ( $<4$ weeks therapy) due to death from IA in 4, death unrelated but with active IA in 1, lost to follow-up in 1, and toxicity in 5 cases.

The voriconazole dose was escalated to $500 \mathrm{mg}$ daily in 4 patients because of inadequate clinical response or radiographic deterioration, without plasma concentration moni- toring. Improvement was seen in 3 cases after the dose was escalated. In one case, the dose was reduced to $200 \mathrm{mg} /$ day because of a severe ECG abnormality and hypoglycemia.

From the 36 evaluable cases, 3 patients $(8.3 \%)$ had a complete response, 16 (44.4\%) had a partial response, and 9 $(25 \%)$ had a stable response at the end of treatment (Table 2). Complete response was only recorded in those with subacute IA. They were treated for 168,118 , and 84 days, respectively. In 2 of these 3 cases, voriconazole was given as primary therapy. Two patients suffered from AIDS and subacute pulmonary IA, and airways invasive disease, respectively. The third one was an immunocompetent patient with posttraumatic carpal bones osteomyelitis. Among the partial responders, 10 of 15 were patients with CPA (Table

Table 2 Response at the End of Therapy, by Site or Type of Disease

\begin{tabular}{|c|c|c|c|c|c|}
\hline \multirow[b]{2}{*}{ Site or Type } & \multirow[b]{2}{*}{ No. of Patients } & \multicolumn{4}{|c|}{ Response to Treatment, no. (\%) } \\
\hline & & Complete & Partial & Stable & Failure \\
\hline Subacute pulmonary & 10 & 1 & 4 & 2 & 3 \\
\hline Paranasal & 4 & - & 2 & 2 & - \\
\hline Osteomyelitis & 3 & 1 & - & 1 & 1 \\
\hline Chronic cavitary pulmonary & 15 & & $10(67)^{*}$ & $2(13)$ & $3(20)$ \\
\hline Total & 36 & $3(8)$ & $16(44)$ & $9(25)$ & $8(22)$ \\
\hline
\end{tabular}

*The best response possible in this group was improved as described in the text. 
Table 3 Response of Subacute IA at the End of Therapy, by Patient Group

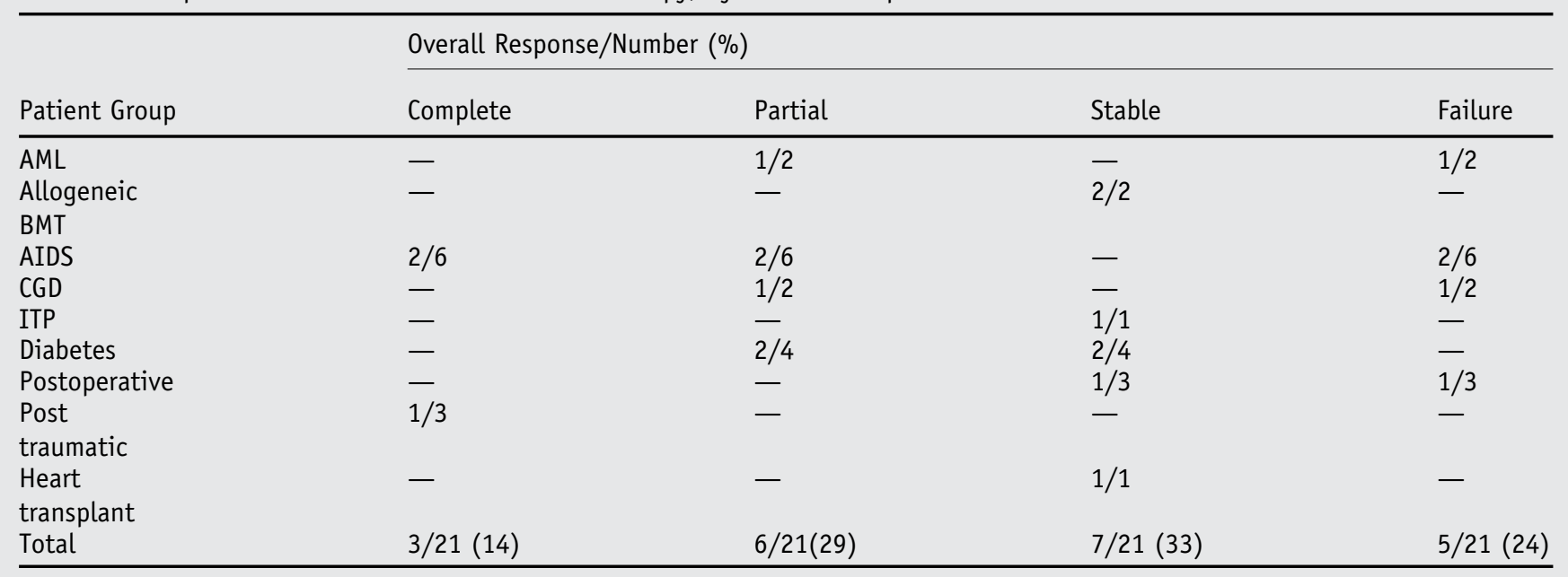

$\mathrm{AML}=$ acute myeloid leukemia; BMT = bone marrow transplant; CGD = chronic granulomatous disease; ITP = idiopathic thrombocytopenic purpura.

2). Thus, among those with sub-acute IA, 9 of $21(43 \%)$ had a favorable response. Eight patients (22\%) failed to respond to therapy after 4 to 182 days (median 47) of treatment. In 3 patients who failed, voriconazole was given as primary therapy, and 4 of the 8 patients who failed were apparently immunocompetent. Of note, 3 of the patients who failed to respond had an extensive local infection (spinal and pulmonary IA in context of relapsed AML and pulmonary and chest wall IA in context of CGD) or haematogenous disseminated disease.

Of the 36 evaluable patients, 11 (31\%) died. Of these, 6 patients had infections that failed to respond to treatment (the duration of voriconazole treatment was 3, 5, 7, 14, 25, and 46 days, respectively), 4 patients were stable, and 1 patient partially responded (end-stage human immunodeficiency virus [HIV] infection with subacute pulmonary aspergillosis). Autopsy was performed in 2 cases, both classified as failures. One patient with CPA and pleural aspergillosis that had been surgically drained died after 3 days of voriconazole, given as a primary treatment, with respiratory failure and multi-organ failure. Autopsy revealed aspergillosis limited to the right lung with no dissemination. The other postallogeneic bone marrow transplant patient with sinus aspergillosis refractory to conventional therapy died of pneumonia after 91 days of voriconazole with stable fungal infection. Lung autopsy revealed interstitial fibrosis compatible with chronic fibrotic graft-versus-host disease, pneumonia, and no evidence of IA.

There were no relapses after a complete or partial response in all cases in which follow-up was available. Improvement was seen during the follow-up period in 5 cases after a stable $(n=3)$ or partial $(n=2)$ response. One patient with CGD and subacute pulmonary IA achieved a partial response following 284 days of voriconazole primary therapy and continued to improve with itraconazole over 12 weeks. Two others improved with itraconazole after a stable response with voriconazole.
Patients with pulmonary aspergillosis had a favorable response of 60\% (15/25) (subacute: 50\% [5/10] - complete and partial, CPA: $66.7 \%$ [10/15] improved), compared with $36 \%$ (4/11) in those with extrapulmonary IA (Table 2). Patients with a sinus infection had a 50\% (2/4) favorable response and $50 \%$ stable response, although in only 2 of the 4 cases there was complementary surgical debridement. In 2 cerebral cases, there was a stable response (after 8 and 63 days of voriconazole, respectively), with a fatal outcome in the third case unrelated to IA. The osteomyelitis cases showed a complete response in one case of posttraumatic wrist infection in an immunocompetent patient, documented with a posttreatment biopsy, a stable response after 17 days on voriconazole in a patient with sternal osteomyelitis following a heart transplant at follow-up after 4 weeks of itraconazole, and failure in the patient with spinal osteomyelitis and AML.

A favorable response was seen in 8 of $16(50 \%)$ immunocompromised patients, compared with 40\% (8/20) among patients with little or no immunocompromising factors (Table 3). Of note, a complete or partial response of $60 \%$ was found in the 6 AIDS cases, all with a definite diagnosis in life.

Better, but statistically insignificant, responses were seen in patients treated with voriconazole as primary versus salvage therapy ( $59 \%$ vs. $43 \%$ ). However, this difference was true only of CPA cases (67\% vs $50 \%)$ and not in the non-CPA cases ( $44 \%$ vs. $42 \%$ ) (Table 4$)$. Among the patients who received voriconazole as salvage therapy, 7 patients improved following prior primary treatment with itraconazole for $11,189,73,>365$ days, and 16 and 18 months, respectively, at doses of $200-600 \mathrm{mg} /$ day. Three salvage patients had stable responses after receiving voriconazole for 63-91 days; one had thoracic wall abscess healing and improvement of pulmonary signs, and the other one had a clinical and histological improvement of sinus infection. 
Table 4 Response at the End of Treatment by Certainty and Kind of Treatment

\begin{tabular}{|c|c|c|c|c|}
\hline \multirow{2}{*}{$\begin{array}{l}\text { Certainty of IA/Kind of } \\
\text { Treatment }\end{array}$} & \multicolumn{4}{|c|}{ Response to Treatment, no. (\%) } \\
\hline & Complete & Partial & Stable & Failure \\
\hline Probable IA & - & - & $1 / 2(50)$ & $1 / 2(50)$ \\
\hline Total & $3 / 21(14.3)$ & $6 / 21(28.6)$ & $7 / 21(33.3)$ & $5 / 21(23.8)$ \\
\hline \multicolumn{5}{|l|}{ Subacute IA cases } \\
\hline Subtotal & $3 / 21(14.3)$ & $6 / 21(28.6)$ & $7 / 21(33.3)$ & $5 / 21(23.8)$ \\
\hline \multicolumn{5}{|l|}{ CPA cases } \\
\hline Primary & & $9 / 13(69 \%)^{*}$ & $2 / 12(16.7)$ & 2/12 (16.7) \\
\hline Salvage & & $1 / 2(50)$ & - & $1 / 2(50)$ \\
\hline Subtotal & & $10 / 15(66.7)$ & $2 / 15(13.3)$ & $3 / 15(20)$ \\
\hline
\end{tabular}

A total of 127 adverse events were reported in 32 patients. Of these, $42(33 \%)$ were attributed by the investigator to voriconazole, the majority of them mild. There were 27 serious adverse events, only 3 of which were attributed to the drug by the investigator. Two of the drug-related severe adverse events were severe increase in liver function tests (LFTs), >7-fold elevation (in 1 of them along with severe arrhythmia leading to death), and the third was severe hypoglycemia (resolved during treatment) along with severe abnormalities in ECG. The most common adverse events were skin reactions, visual disturbances (12/32), and abnormal liver function tests (13/32). There was no relationship between the emergence and severity of adverse events and duration of treatment. Of the 6 cases with early discontinuation due to adverse events, 5 were hepatic and in the first 4 weeks, (3 severe, 2 moderate), and one due to nausea and vomiting after 6 weeks. Two types of skin reaction were noted: rash $(n=6)$, photosensitivity $(n=3)$, and cheilitis ( $n$ $=5$ ). None of the patients discontinued treatment because of a skin reaction. ${ }^{25,26}$ Visual disturbances such as enhanced light perception, photophobia, color vision changes, blurred vision, and wavy lines on television or on going to sleep were often reported (12 patients [37.5\%]). No intervention was required and they resolved without discontinuation of the drug. The LFT abnormalities included elevation in individual enzymes of bilirubin alone or together. Most abnormal LFTs resolved without dose adjustment during treatment, but in 5 cases resolved after the end of treatment.

\section{DISCUSSION}

Our patient population comprised those with subacute IA and CPA. Several large prospective studies of acute IA have been published. ${ }^{8,24}$ Almost all the IA cases had proven disease. Many of the cases had few or no immunocompromising factors, and some were very complicated. Overall, there was a $43 \%$ favorable response for subacute IA cases (complete and partial) and a $24 \%$ failure rate. In salvage IA cases the response rate was $41.7 \%$. These response rates for IA are comparable with the reported efficacy of other recent prospective studies of voriconazole in acute $\mathrm{IA}^{23,24}$ and consistent with previous experience with itraconazole in IA. ${ }^{13,16,17}$ Surgery was performed only occasionally and did not have any meaningful impact on outcome, even in cases where surgical debridement and drainage is considered to be of primary importance, such as in sinus infections.

The $67 \%$ favorable (complete and partial) response in the 6 AIDS cases is the highest ever reported. Of note, all were definite cases with antemortem diagnosis. All patients were recruited in the pre-HAART (highly active anti-retroviral therapy) era; therefore, an impact of HAART, through immune restoration and pathogen-specific immunity, is excluded. No data about anti-retroviral therapy, CD4 cell count or viral load changes were available. However, all had an end-stage HIV infection with multiple prior or concurrent opportunistic infections. Despite the low reported incidence of IA in AIDS patients $(2.1 \%)$, the mortality from available case reports is high, typically $>80 \% .^{27}$ Of our patients, 1 died of aspergillosis after a short course of 7 days salvage therapy of voriconazole and another 1 of haemoptysis due to IA after 2 weeks of primary therapy with voriconazole (33\% mortality). Among the 93 cases reported in the literature with definite diagnosis of IA in AIDS patients, the diagnosis was established or confirmed by autopsy in $70 \%$, and $35 \%$ had no antermortem test suggestive of aspergillosis.

We report here the only prospective study of CPA. Prospective therapeutic studies of aspergilloma have also been completed. ${ }^{17,18,28,29}$ In our CPA cases, there was an $80 \%$ response rate (improved and stable), all but 2 of whom were primary cases. Most of these were improved responses, as opposed to stable, with both clinical and radiological improvement. Studies of CPA are associated 
with many inherent problems. Diagnostic criteria have been proposed ${ }^{6}$ but not yet applied in a prospective study. The evaluation of response is difficult, because nonspecific clinical markers such as weight gain and improved energy levels are the earliest and definite indicators of response. Response occurs in the first 8 weeks of treatment, with reduction of inflammatory markers, Aspergillus precipitin titers, and improvement of inflammation surrounding cavities and reduction of cavity sizes requiring many months. ${ }^{6}$ Itraconazole, as a primary therapy, is effective in $45 \%$ of CPA cases. ${ }^{6}$ Our experience with relapse on stopping therapy indicates that CPA is an incurable disease, and either lifelong or prolonged periods of therapy are required.

Voriconazole has an acceptable tolerability. The most common adverse events observed included transient visual disturbances, hepatotoxicity, and skin reaction. Monitoring of hepatic function is important during voriconazole therapy, as 5 patients developed severe or moderate elevation in LFTs during treatment. It is our view that monitoring of plasma concentrations to ensure appropriate exposure is appropriate, especially in those with much concomitant medication or features suggestive of hepatic dysfunction, but this remains to be clinically validated.

Given its desirable broad spectrum and the excellent results of in vitro and animal studies, voriconazole is a potentially appropriate first-line treatment for life-threatening fungal infections and is now licensed as a primary therapy for acute IA. Treatment of chronic aspergillosis requires long-term therapy, with an acceptable toxicity profile, often for months or even years, and the use of an orally administered regimen, as the only realistic option. The results of our data show that voriconazole is a safe and effective treatment as primary or salvage therapy in chronic forms of IA, irrespective of the immunological status of the patients.

\section{ACKNOWLEDGMENTS}

We are indebted to colleagues who enrolled patients in the study including from Germany: H Breithaupt, (Giesson), W. Mathiesson (Dresden); Italy: G. Torlontano (Perugia), C. Viscoli (Genoa); Netherlands: A. Hoepelman (Utrecht), B-J. Kullberg (Nijmegen), S. Van der Geest (Maastricht); Switzerland: M. Glauser; United Kingdom: R. Hay (Belfast), and to Dr. Elizabeth Johnson, from the HPA Mycology Reference Laboratory who analyzed the serum samples for galactomannan. The study was supported by Pfizer Ltd, Sandwich UK.

\section{References}

1. Denning DW. Invasive aspergillosis. Clin Infect Dis. 1998;26:781805.

2. Hope WW, Walsh TJ, Denning DW. The invasive and saprophytic syndromes due to Aspergillus spp. Med Mycol. 2005;43(Suppl 1): S207-S238.
3. Denning DW. Chronic forms of pulmonary aspergillosis. Clin Microb Infect. 2001;7(Suppl 2):25-31.

4. Crosdale D, Poulton K, Ollier W, Thomson W, Denning DW. Mannose binding lectin gene polymorphisms as a susceptibility factor for chronic necrotising pulmonary aspergillosis. J Infect Dis. 2001;184: 653-656.

5. Sambatakou H, Pravica V, Hutchinson I, Denning DW. Cytokine profiling of pulmonary aspergillosis. Int J Immunogenetics. 2006; In press.

6. Denning DW, Riniotis K, Dobrashian R, Sambatakou H. Chronic cavitary and fibrosing pulmonary and pleural aspergillosis: Case series, proposed nomenclature and review. Clin Infect Dis. 2003; 37(Suppl 3):S265-S280.

7. Patterson TF, Kirkpatrick WR, White M, et al. Invasive aspergillosis. Disease spectrum, treatment practices, and outcomes. I3 Aspergillus Study Group. Medicine (Baltimore). 2000;79:250-260.

8. Bowden R, Choudrasekar P, White MH, et al. A double-blind, randomized controlled trial of amphotericin $\mathrm{B}$ colloidal dispersion versus amphotericin B for treatment of invasive aspergillosis in immunocompromised patients. Clin Infect Dis. 2002;35:359-366.

9. Moore CB, Sayers N, Mosquero J, Slaven J, Denning DW. Antifungal drug resistance in Aspergillus. J Infect. 2000;41:203-220.

10. Sutton DA, Sanche SE, Revankar SG, et al. In vitro amphotericin B resistance in clinical isolates of Aspergillus terreus, with a head-tohead comparison to voriconazole. J Clin Microbiol. 1999;37:23432345.

11. Tritz D, Woods G. Fatal disseminated infection with Aspergillus terreus in the immunocompromised patient. Clin Infect Dis. 1993;16: 118-122.

12. Maertens J, Raad I, Petrikkos G, et al. A multicenter, open, noncomparative study to estimate the safety, tolerability and efficacy of caspofungin (MK-0991) in the treatment of invasive Aspergillus infections in adults who are refractory to or intolerant of amphotericin B, lipid formulations of amphotericin B or azoles. Clin Infect Dis. 2004;39: 1563-1571.

13. Denning DW, Lee JY, Hostetler JS, et al. NIAID Mycoses Study Group multicenter trial of oral itraconazole therapy of invasive aspergillosis. Am J Med. 1994;97:135-144.

14. Stevens DA, Lee JY. Analysis of compassionate use itraconazole for invasive aspergillosis by the NIAID Mycoses Study Group criteria. Arch Intern Med. 1997;157:1857-1862.

15. Denning DW, Tucker RM, Hanson LH, Stevens DA. Treatment of invasive aspergillosis with itraconazole. Am J Med. 1989;86:791-800.

16. Viviani MA, Tortorano AM, Woestenborghs R. Cauwenbergh G. Experience with itraconazole in deep mycoses in northern Italy. Mykosen. 1987;30:233-244.

17. Dupont B. Itraconazole therapy in aspergillosis: study in 49 patients. J Am Acad Dermatol. 1990;23:607-614.

18. DeBeule K, DeDoncker P, Cauwenbergh G, et al. The treatment of aspergillosis and aspergilloma with itraconazole: clinical results of an open international study (1982-1987). Mycoses. 1988;31:476-485.

19. Mosquera J Denning DW. Azole cross-resistance in Aspergillus fumigatus. Antimicrob Agents Chemother. 2002;46:556-557.

20. Martin MV, Yates J, Hitchcock CA. Comparison of voriconazole (UK-109,496) and itraconazole in prevention and treatment of Aspergillus fumigatus endocarditis in guinea pigs. Antimicrob Agents Chemother. 1997;41:13-16.

21. Murphy M, Bernard EM, Ishimaru T, Armstrong D. Activity of voriconazole (UK-109,496) against clinical isolates of Aspergillus species and its effectiveness in an experimental model of invasive pulmonary aspergillosis. Antimicrob Agents Chemother. 1997;41:696-698.

22. Ghannoum MA, Kuhn DM. Voriconazole-better chances for patients with invasive mycoses. Eur J Med Res. 2002;7:242-256.

23. Denning DW, Ribaud P, Milpied N, et al. The efficacy and safety of voriconazole in the treatment of acute invasive aspergillosis. Clin Infect Dis. 2002;34:563-571. 
24. Herbrecht R, Denning DW, Patterson TF, et al. Randomised comparison of voriconazole and amphotericin B in primary therapy of invasive aspergillosis. N Engl J Med. 2002;347:408-415.

25. Khoo S, Denning DW. Aspergillus infection in the acquired immune deficiency syndrome. Clin Infect Dis. 1994;19(Suppl 1):541548.

26. Libanore M, Prini E, Mazzetti M, et al. Invasive aspergillosis in Italian AIDS patients. Infection. 2002;30:341-345.
27. Mylonakis E, Barlam TF, Flanigan T, Rich JD. Pulmonary aspergillosis and invasive disease in AIDS. Review of 342 cases. Chest. 1998;114:251-262.

28. Lebeau B, Pelloux H, Pinel C, et al. Itraconazole in the treatment of aspergillosis: a study of 16 cases. Mycoses. 1994;37:171-179.

29. Campbell JH, Winter JH, Richardson MD, Shankland GS, Banham SW. Treatment of pulmonary aspergilloma with itraconazole. Thorax. 1991;46:839-841. 